\title{
Next generation sequencing and array-based comparative genomic hybridization for molecular diagnosis of pediatric endocrine disorders
}

\author{
Maki Fukami, MD, \\ Mami Miyado, PhD
}

Department of Molecular Endocrinology, National Research Institute for Child Health and Development, Tokyo, Japan
Received: 23 May, 2017

Accepted: 17 June, 2017

Address for correspondence:

Maki Fukami, MD

Department of Molecular Endocrinology, National Research Institute for Child Health and Development, 2-10-1 Okura, Setagaya, Tokyo 1578535, Japan

Tel: +81-3-5494-7025

Fax: +81-3-5494-7026

E-mail: fukami-m@ncchd.go.jp

https://orcid.org/0000-0001-99714035
Next-generation sequencing (NGS) and array-based comparative genomic hybridization (array CGH) have enabled us to perform high-throughput mutation screening and genome-wide copy number analysis, respectively. These methods can be used for molecular diagnosis of pediatric endocrine disorders. NGS has determined the frequency and phenotypic variation of mutations in several disease-associated genes. Furthermore, whole exome analysis using NGS has successfully identified several novel causative genes for endocrine disorders. Array $\mathrm{CGH}$ is currently used as the standard procedure for molecular cytogenetic analysis. Array CGH can detect various submicroscopic genomic rearrangements involving exons or enhancers of disease-associated genes. This review introduces some examples of the use of NGS and array CGH for the molecular diagnosis of pediatric endocrine disorders.

Keywords: Next-generation sequencer, Mutation, Comparative genomic hybridization, DNA Copy-number variations, Diagnosis

\section{Introduction}

Single gene mutations and submicroscopic chromosomal rearrangements account for a certain percentage of the etiology of pediatric endocrine disorders ${ }^{1-3)}$. Identification of such molecular defects is beneficial for patients, because molecular diagnosis often allows better clinical management and accurate genetic counseling ${ }^{4,5)}$. Furthermore, identification of the genetic causes of congenital endocrine disorders provides advances in medical research.

Recently, various molecular technologies have been developed to identify genetic and genomic alterations in humans. These technologies include next generation sequencing (NGS) that can detect nucleotide substitutions and small insertions/deletions (indels) ${ }^{6-8)}$, and array-based comparative genomic hybridization (array CGH) that identifies copy-number variations (CNVs) in the genome ${ }^{9-12)}$. NGS and array CGH enable us to perform systematic molecular analyses for multiple clinical samples. This review introduces some examples of the use of NGS and array CGH for the molecular diagnosis of pediatric endocrine disorders.

\section{Molecular diagnosis of pediatric endocrine disorders using NGS}

NGS is a powerful tool for high-throughput mutation analysis ${ }^{6-8)}$. This method is particularly useful to elucidate the genetic causes of Mendelian disorders with multiple causative genes. In our recent studies ${ }^{13-16)}$, we performed NGS analyses for several samples of patients with pediatric endocrine disorders (Fig. 1). For example, we investigated the genetic causes of hypogonadotropic hypogonadism $(\mathrm{HH})$, a rare condition caused by monoallelic, biallelic, or oligogenic mutations in more than 20 genes ${ }^{13,16)}$. We created a custom-made NGS 
panel that covers most known causative genes for HH. DNA samples obtained from 58 patients with $\mathrm{HH}$ with and without additional clinical features were analyzed ${ }^{13)}$. As a result, possible pathogenic mutations were identified in 13 of the 58 patients. Mutations in FGFR1 and CHD7 accounted for the majority of the molecular defects in our patients ${ }^{13)}$. We identified an SOX 3 polyalanine deletion, a rare mutation known to cause pituitary dysfunction ${ }^{17)}$, in a patient with normosmic isolated $\mathrm{HH}(\mathrm{IHH})^{13)}$. This finding expanded the phenotypic spectrum of SOX3 polyalanine deletion to include $\mathrm{HH}$ without other pituitary hormone deficiency. Likewise, we detected a WDR11 splice-site mutation in a patient with combined pituitary hormone deficiency (CPHD) ${ }^{13)}$. These data indicated that haploinsufficiency of WDR11, a gene that has recently been implicated in normosmic IHH and Kallmann syndrome $(\mathrm{HH}$ with anosmia) $)^{18)}$, can also underlie CPHD.

In another study, we analyzed 62 patients with nonsyndromic hypospadias (hypospadias without additional clinical features), a common anomaly in boys ${ }^{15)}$. We created an NGS panel covering the coding exons of 25 known disease-associated genes, and detected mutations in $A R, N R 5 A 1, B N C 2$ and other genes in seven cases ${ }^{15)}$. These data implied that more than $10 \%$ of cases with nonsyndromic hypospadias arise from mutations in known causative genes. Importantly, one patient carried probably damaging mutations in multiple genes ${ }^{15)}$, suggesting the possible oligogenicity of nonsyndromic hypospadias.

The abovementioned results provided evidence that mutation screening using NGS is helpful to clarify the contribution of each causative gene to the etiology of polygenic disorders. In addition, NGS can provide critical information about phenotypic variations of each monogenic abnormality.

\section{Identification of novel genetic causes of pediatric endocrine disorders by NGS}

NGS can also be used for systematic mutation screening of all

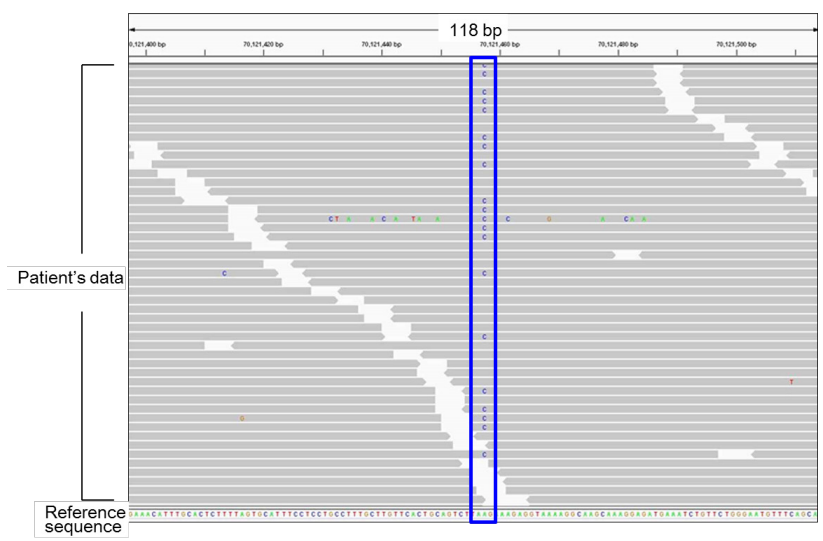

Fig. 1. Representative results of next generation sequencing. Each gray bar denotes a 100-bp DNA fragment obtained from a patient's sample. Substituted nucleotides are highlighted by colored letters. This patient carries a heterozygous A to $C$ substitution (the blue box). All other substitutions in this figure are likely to be sequence errors. known protein-coding genes (whole exome analysis) ${ }^{8,19)}$. To date, whole exome analysis has successfully detected several novel causative genes for human disorders. For example, in 2013, Abreu et al. ${ }^{20)}$ performed whole exome analysis of 15 patients with central precocious puberty (CPP) of unknown etiology. NGS analysis of the patients and their unaffected family members identified MKRN3 mutations cosegregated with the disease. Subsequent studies confirmed that MKRN3 mutations constitute one of the major genetic causes of $\mathrm{CPP}^{21-23)}$.

Similarly, whole exome analysis helped to identify a novel cause of disorders of sex development (DSD). Bashamboo et al., Baetens et al., and our group revealed that an identical missense substitution in NR5A1, p.R92W, induces testicular development in genetic females ${ }^{24-26)}$. This finding provided the first indication that specific missense mutations in a single gene can underlie 46,XX testicular/ovotesticular DSD. Since the p.R92W mutation in Nr5al did not induce testicular formation in female mice ${ }^{27)}$, it appears that NR5A1 plays a species-specific role in sexual development.

\section{Array CGH-based copy-number analysis for molecular diagnosis of pediatric endocrine disorders}

Array CGH can screen CNVs in the entire genome in a single assay ${ }^{9-12)}$. This method is currently used as the standard procedure for molecular cytogenetic analysis. Indeed, array CGH has higher resolution than conventional G-banding ${ }^{12)}$, although array CGH cannot detect copy-number neutral translocations and inversions. To date, it is known that CNVs account for a significant fraction of the etiology of Mendelian disorders ${ }^{12,28)}$. We have performed array CGH for several patients with pediatric endocrine disorders ${ }^{14,29)}$. We analyzed DNA samples obtained from 24 patients with 46,XY DSD of unknown etiology, and identified submicroscopic deletions in 3 patients ${ }^{29)}$. These results suggested that submicroscopic CNVs represent important genetic causes of 46,XY DSD. These deletions were predicted to induce undermasculinized external genitalia by eliminating exons or cis-regulatory elements

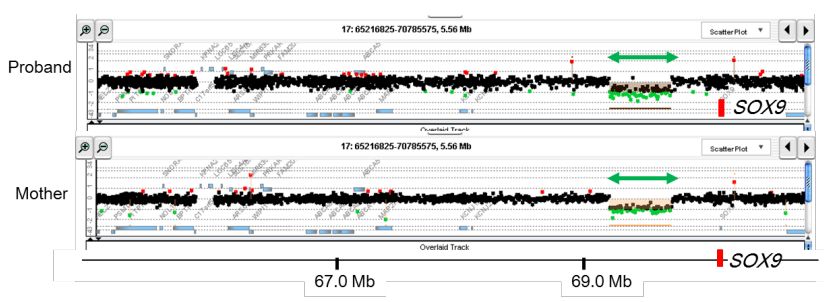

Fig. 2. Representative results of array-based comparative genomic hybridization. Submicroscopic deletion identified in a patient with $46, X Y$ disorders of sex development is shown. This deletion likely encompasses a cis-acting enhancer(s) of SOX9 ${ }^{14)}$. The deletion was shared by the patient's mother. The black, red, and green dots denote signals indicative of the normal, the increased $(>+0.5)$ and the decreased $(<-1.0)$ copy-numbers, respectively. Green arrows depict deleted regions. Genomic positions refer to the Human Genome (hg19, build 37). 
of genes involved in sex development. Notably, one patient with complete 46,XY gonadal dysgenesis was found to carry a microdeletion in a far upstream region of SOX9 (Fig. 2) ${ }^{14)}$, indicating that a testis-specific enhancer(s) of $S O X 9$ is located within this deleted region.

Similarly, we utilized array CGH to characterize CNVs in the short arm pseudoautosomal region of sex chromosomes (PAR1) associated with idiopathic short stature or Leri-Weill dyschondrosteosis (short stature accompanied by Madelung deformity in the forearm $)^{30-32)}$. It is known that CNVs in PAR1 encompassing SHOX and/or its putative enhancers

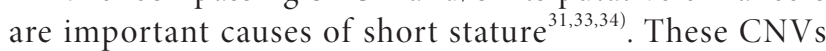
were predicted to affect skeletal growth by reducing SHOX expression levels in chondrocytes ${ }^{30,311}$. We found that CNVs in PAR1 are highly variable in their sizes and positions ${ }^{30-32)}$. Breakpoint characterization of the CNVs suggested that various mechanisms such as non-allelic homologous recombination and non-homologous end joining, and possibly replication errors as well, are involved in these abnormalities ${ }^{30,31,34,35)}$. Thus, SHOX abnormality represents a model of human genomic disorders.

\section{Identification of novel genetic causes of pediatric endocrine disorders by array CGH}

Array CGH has also identified novel genomic abnormalities associated with pediatric endocrine disorders. For example, we performed array CGH analysis for patients with hereditary gynecomastia (aromatase excess syndrome), a rare autosomal dominant disorder characterized by estrogen overproduction due to excessive enzymatic activity of aromatase ${ }^{36-39)}$. We found that these patients have submicroscopic CNVs in the upstream region of the aromatase gene $(C Y P 19 A 1)^{36-39)}$. These CNVs included microduplications encompassing CYP19A1 promoters and microdeletions that lead to aberrant fusion between the coding exons of CYP19A1 and promoters of widely expressed neighboring genes ${ }^{38)}$. These data suggested that hereditary gynecomastia is caused by submicroscopic genomic rearrangements leading to CYP19A1 overexpression ${ }^{38}$. Most importantly, our results indicated for the first time that a tandem duplication involving physiological promoters can underlie gene overexpression and resultant congenital disorders ${ }^{36,38)}$.

\section{Ethical issues associated with the use of NGS and array CGH}

Although the use of NGS and array CGH is highly beneficial for patients with pediatric endocrine disorders as well as other genetic diseases, these new technologies also raise ethical issues that are not associated with conventional molecular analyses. For example, whole exome analysis can detect mutations that are not related with the patient's present phenotype ${ }^{40,411}$. Some of these unexpected genetic alterations may have a potential to cause some serious disorders later in life. In addition, NGS and array CGH frequently detect molecular alterations of unknown pathogenicity ${ }^{5}$. There is debate as to which and how the findings of systematic molecular analyses should be reported to the patients. The American College of Medical Genetics and Genomics and other organizations have published recommendations for the management of data obtained from NGS analysis ${ }^{41-43)}$.

In addition, NGS and array CGH can be applied to prenatal diagnosis ${ }^{44,45}$. For example, NGS is used for noninvasive prenatal testing for chromosomal aneuploidy ${ }^{45}$. NGS and array CGH have broad applicability in the prenatal molecular diagnosis of both Mendelian disorders and multifactorial conditions ${ }^{5}$. However, the benefits, limitations, and ethical issues of these technologies need to be discussed.

\section{Future implications}

NGS and array CGH are beneficial both for clinical practice and for research on pediatric endocrinology. In particular, since the cost of NGS is continuously decreasing, the utility of this method will be further enhanced in the future. Moreover, NGS has additional applications such as characterization of gut microbial flora in individuals with specific conditions ${ }^{46,47}$. The use of NGS and array CGH will provide further information about the causative mechanisms of pediatric endocrine disorders.

\section{Conflict of interest}

No potential conflict of interest relevant to this article was reported.

\section{Acknowledgments}

This study was supported by the Grants-in-Aid from the Japan Society for the Promotion of Science; and by the Grants from the Ministry of Health, Labor and Welfare, the Japan Agency for Medical Research and Development, the National Center for Child Health and Development and the Takeda Foundation.

\section{References}

1. Lee C, Iafrate AJ, Brothman AR. Copy number variations and clinical cytogenetic diagnosis of constitutional disorders. Nat Genet 2007;39(7 Suppl):S48-54.

2. Buonocore F, Achermann JC. Human sex development: targeted technologies to improve diagnosis. Genome Biol 2016;17:257.

3. Eggers S, Sadedin S, van den Bergen JA, Robevska G, Ohnesorg T, Hewitt J, et al. Disorders of sex development: insights from targeted gene sequencing of a large international patient cohort. Genome Biol 2016;17:243.

4. Burke W. Genetic testing. N Engl J Med 2002;347:1867-75. 
5. Katsanis $\mathrm{SH}$, Katsanis N. Molecular genetic testing and the future of clinical genomics. Nat Rev Genet 2013;14:415-26.

6. Smith DR, Quinlan AR, Peckham HE, Makowsky K, Tao W, Woolf B, et al. Rapid whole-genome mutational profiling using next-generation sequencing technologies. Genome Res 2008;18:1638-42.

7. Shendure J, Ji H. Next-generation DNA sequencing. Nat Biotechnol 2008;26:1135-45.

8. Metzker ML. Sequencing technologies: the next generation. Nat Rev Genet 2010;11:31-46.

9. Pinkel D, Segraves R, Sudar D, Clark S, Poole I, Kowbel D, et al. High resolution analysis of DNA copy number variation using comparative genomic hybridization to microarrays. Nat Genet 1998;20:207-11.

10. Vissers LE, de Vries BB, Osoegawa K, Janssen IM, Feuth T, Choy CO, et al. Array-based comparative genomic hybridization for the genomewide detection of submicroscopic chromosomal abnormalities. Am J Hum Genet 2003;73:1261-70.

11. Feuk L, Carson AR, Scherer SW. Structural variation in the human genome. Nat Rev Genet 2006;7:85-97.

12. Miller DT, Adam MP, Aradhya S, Biesecker LG, Brothman AR, Carter NP, et al. Consensus statement: chromosomal microarray is a first-tier clinical diagnostic test for individuals with developmental disabilities or congenital anomalies. Am J Hum Genet 2010;86:749-64.

13. Izumi Y, Suzuki E, Kanzaki S, Yatsuga S, Kinjo S, Igarashi M, et al. Genome-wide copy number analysis and systematic mutation screening in 58 patients with hypogonadotropic hypogonadism. Fertil Steril 2014;102:1130-6.e3.

14. Katoh-Fukui Y, Igarashi M, Nagasaki K, Horikawa R, Nagai $\mathrm{T}$, Tsuchiya T, et al. Testicular dysgenesis/regression without campomelic dysplasia in patients carrying missense mutations and upstream deletion of SOX9. Mol Genet Genomic Med 2015;3:550-7.

15. Kon M, Suzuki E, Dung VC, Hasegawa Y, Mitsui T, Muroya $\mathrm{K}$, et al. Molecular basis of non-syndromic hypospadias: systematic mutation screening and genome-wide copynumber analysis of 62 patients. Hum Reprod 2015;30:499506.

16. Fukami M, Suzuki E, Izumi Y, Torii T, Narumi S, Igarashi M, et al. Paradoxical gain-of-function mutant of the G-proteincoupled receptor PROKR2 promotes early puberty. J Cell Mol Med 2017 Mar 24 [Epub]. https://doi.org/10.1111/ jcmm.13146.

17. Alatzoglou KS, Kelberman D, Cowell CT, Palmer R, Arnhold IJ, Melo ME, et al. Increased transactivation associated with SOX3 polyalanine tract deletion in a patient with hypopituitarism. J Clin Endocrinol Metab 2011;96:E685-90.

18. Kim HG, Ahn JW, Kurth I, Ullmann R, Kim HT, Kulharya A, et al. WDR11, a WD protein that interacts with transcription factor EMX1, is mutated in idiopathic hypogonadotropic hypogonadism and Kallmann syndrome. Am J Hum Genet 2010;87:465-79.

19. Bamshad MJ, Ng SB, Bigham AW, Tabor HK, Emond
MJ, Nickerson DA, et al. Exome sequencing as a tool for Mendelian disease gene discovery. Nat Rev Genet 2011;12:745-55.

20. Abreu AP, Dauber A, Macedo DB, Noel SD, Brito VN, Gill JC, et al. Central precocious puberty caused by mutations in the imprinted gene MKRN3. N Engl J Med 2013;368:246775 .

21. Settas N, Dacou-Voutetakis C, Karantza M, KanakaGantenbein C, Chrousos GP, Voutetakis A. Central precocious puberty in a girl and early puberty in her brother caused by a novel mutation in the MKRN3 gene. J Clin Endocrinol Metab 2014;99:E647-51.

22. Shin YL. An update on the genetic causes of central precocious puberty. Ann Pediatr Endocrinol Metab 2016;21:66-9.

23. Christoforidis A, Skordis N, Fanis P, Dimitriadou M, Sevastidou M, Phelan MM, et al. A novel MKRN3 nonsense mutation causing familial central precocious puberty. Endocrine 2017;56:446-9.

24. Bashamboo A, Donohoue PA, Vilain E, Rojo S, Calvel P, Seneviratne SN, et al. A recurrent p.Arg92Trp variant in steroidogenic factor-1 (NR5A1) can act as a molecular switch in human sex development. Hum Mol Genet 2016;25:3446-53.

25. Baetens D, Stoop H, Peelman F, Todeschini AL, Rosseel T, Coppieters F, et al. NR5A1 is a novel disease gene for 46,XX testicular and ovotesticular disorders of sex development. Genet Med 2017;19:367-76.

26. Igarashi M, Takasawa K, Hakoda A, Kanno J, Takada S, Miyado M, et al. Identical NR5A1 missense mutations in two unrelated 46,XX individuals with testicular tissues. Hum Mutat 2017;38:39-42.

27. Miyado M, Inui M, Igarashi M, Katoh-Fukui Y, Takasawa K, Hakoda A, et al. The p.R92W variant of NR5A1/ Nr5al induces testicular development of 46,XX gonads in humans, but not in mice: phenotypic comparison of human patients and mutation-induced mice. Biol Sex Differ 2016;7:56.

28. Beckmann JS, Estivill X, Antonarakis SE. Copy number variants and genetic traits: closer to the resolution of phenotypic to genotypic variability. Nat Rev Genet 2007;8:639-46.

29. Igarashi M, Dung VC, Suzuki E, Ida S, Nakacho M, Nakabayashi K, et al. Cryptic genomic rearrangements in three patients with 46,XY disorders of sex development. PLoS One 2013;8:e68194.

30. Fukami M, Naiki Y, Muroya K, Hamajima T, Soneda S, Horikawa R, et al. Rare pseudoautosomal copy-number variations involving SHOX and/or its flanking regions in individuals with and without short stature. J Hum Genet. 2015;60:553-6.

31. Fukami M, Seki A, Ogata T. SHOX haploinsufficiency as a cause of syndromic and nonsyndromic short stature. Mol Syndromol 2016;7:3-11.

32. Shima H, Tanaka T, Kamimaki T, Dateki S, Muroya K, Horikawa R, et al. Systematic molecular analyses of SHOX 
in Japanese patients with idiopathic short stature and LeriWeill dyschondrosteosis. J Hum Genet 2016;61:585-91.

33. Benito-Sanz S, Barroso E, Heine-Suñer D, Hisado-Oliva A, Romanelli V, Rosell J, et al. Clinical and molecular evaluation of SHOX/PAR1 duplications in Leri-Weill dyschondrosteosis (LWD) and idiopathic short stature (ISS). J Clin Endocrinol Metab 2011;96:E404-12.

34. Benito-Sanz S, Royo JL, Barroso E, Paumard-Hernández B, Barreda-Bonis AC, Liu P, et al. Identification of the first recurrent PAR1 deletion in Léri-Weill dyschondrosteosis and idiopathic short stature reveals the presence of a novel SHOX enhancer. J Med Genet 2012;49:442-50.

35. Fukami M, Kato F, Tajima T, Yokoya S, Ogata T. Transactivation function of an approximately 800-bp evolutionarily conserved sequence at the SHOX 3 ' region: implication for the downstream enhancer. Am J Hum Genet 2006;78:16770 .

36. Fukami M, Shozu M, Soneda S, Kato F, Inagaki A, Takagi H, et al. Aromatase excess syndrome: identification of cryptic duplications and deletions leading to gain of function of CYP19A1 and assessment of phenotypic determinants. J Clin Endocrinol Metab 2011;96:E1035-43.

37. Fukami M, Tsuchiya T, Vollbach H, Brown KA, Abe S, Ohtsu $\mathrm{S}$, et al. Genomic basis of aromatase excess syndrome: recombination- and replication-mediated rearrangements leading to CYP19A1 overexpression. J Clin Endocrinol Metab 2013;98:E2013-21.

38. Fukami M, Miyado M, Nagasaki K, Shozu M, Ogata T. Aromatase excess syndrome: a rare autosomal dominant disorder leading to pre- or peri-pubertal onset gynecomastia. Pediatr Endocrinol Rev 2014;11:298-305.

39. Shihara D, Miyado M, Nakabayashi K, Shozu M, Ogata T,
Nagasaki K, et al. Aromatase excess syndrome in a family with upstream deletion of CYP19A1. Clin Endocrinol (Oxf) 2014;81:314-6.

40. Vasli N, Böhm J, Le Gras S, Muller J, Pizot C, Jost B, et al. Next generation sequencing for molecular diagnosis of neuromuscular diseases. Acta Neuropathol 2012;124:27383.

41. Green RC, Berg JS, Grody WW, Kalia SS, Korf BR, Martin CL, et al. ACMG recommendations for reporting of incidental findings in clinical exome and genome sequencing. Genet Med 2013;15:565-74.

42. Richards S, Aziz N, Bale S, Bick D, Das S, Gastier-Foster J, et al. Standards and guidelines for the interpretation of sequence variants: a joint consensus recommendation of the American College of Medical Genetics and Genomics and the Association for Molecular Pathology. Genet Med 2015; 17:405-24.

43. Matthijs G, Souche E, Alders M, Corveleyn A, Eck S, Feenstra I, et al. Guidelines for diagnostic next-generation sequencing. Eur J Hum Genet 2016;24:2-5.

44. Wapner RJ, Martin CL, Levy B, Ballif BC, Eng CM, Zachary JM, et al. Chromosomal microarray versus karyotyping for prenatal diagnosis. N Engl J Med 2012;367:2175-84.

45. Benn P, Cuckle H, Pergament E. Non-invasive prenatal testing for aneuploidy: current status and future prospects. Ultrasound Obstet Gynecol 2013;42:15-33.

46. Marrs T, Flohr C. The role of skin and gut microbiota in the development of atopic eczema. Br J Dermatol 2016;175 Suppl 2:13-8.

47. Ma L, Xie Y, Han Z, Giesy JP, Zhang X. Responses of earthworms and microbial communities in their guts to Triclosan. Chemosphere 2017;168:1194-202. 